\title{
Two-dimensional laser-induced incandescence for soot volume fraction measurements: issues in quantification due to laser beam focusing
}

\author{
Manu Mannazhi ${ }^{1}$ (D) Per-Erik Bengtsson ${ }^{1}$
}

Received: 22 July 2020 / Accepted: 26 October 2020 / Published online: 16 November 2020

(C) The Author(s) 2020

\begin{abstract}
Two-dimensional laser-induced incandescence (LII) measurements usually involve the use of a cylindrical lens to illuminate the planar region of interest. This creates a varying laser fluence and sheet width in the imaged flame region which could lead to large uncertainties in the quantification of the 2D LII signals into soot volume fraction distributions. To investigate these effects, 2D LII measurements using a wide range of laser pulse energies were performed on a premixed flat ethylene-air flame while employing a cylindrical lens to focus the laser sheet. Using shorter focal length of the focusing lens resulted in larger variation of the LII signal profiles across the flame. A heat - and - mass - transfer - based LII model was also used to simulate the measurements and good agreement was found. The ratio between focal length (FL) and image length (IL) was introduced as a useful parameter for estimating the bias in estimated soot volume fractions across the flame. The general recommendation is to maximize this FL/IL ratio in an experiment, which in practice means the use of a long focal length lens. Furthermore, the best choices of laser fluence and detection gate width are discussed based on results from these simulations.
\end{abstract}

\section{Introduction}

Laser-induced incandescence (LII) has been one of the most prominent laser diagnostic techniques for performing quantitative two-dimensional (2D) soot measurements in the past few decades $[1,2]$. In this technique, soot particles are heated up to high temperatures $(\sim 4000 \mathrm{~K})$ using high-energy laser pulses. These laser - heated soot particles emit enhanced Planck radiation with respect to the flame soot, and this enhanced radiation is called the LII signal. The LII signal has been found to be proportional to soot volume fraction $\left(f_{v}\right)$, to a first approximation [3], which can be calibrated to absolute values using, for example, extinction measurements [4]. Although LII appears to be a simple technique, there are several challenges in performing accurate quantitative 2D LII measurements. As soot is a strong absorber, the attenuation of the laser beam along the beam

Manu Mannazhi

manu.mannazhi@forbrf.lth.se

Per-Erik Bengtsson

per-erik.bengtsson@forbrf.lth.se

1 Division of Combustion Physics, Department of Physics, Box 118, Lund University, SE-22100 Lund, Sweden propagation direction as well as LII signal trapping from the measurement region to the detector are potential problems $[5,6]$. Another problem often appears in pressurised systems where optical windows are used, where soot contamination commonly appears on the detection window [7]. Furthermore, as combustion environments have strong temperature and density gradients, beam steering may also lower the accuracy in soot volume fraction measurements in practical applications [5, 6].

One problem which occasionally gets overlooked in $2 \mathrm{D}$ LII measurements is the issue of varying laser sheet width in the imaged region along the beam propagation direction caused due to the laser sheet focusing optics. One way to mitigate this problem is to use a very long focal length lens [8] or a combination of lenses $[9,10]$. Another possibility is to collimate the laser sheet both in the height and thickness directions instead of using a cylindrical lens to focus the laser sheet [11], but at the expense of a lower spatial resolution orthogonal to the beam sheet. Often however, there is an expectation that the effect of a varying laser sheet width leads to less uncertainty than other uncertainties in the LII measurements. For example, the known uncertainty in absorption efficiency of soot is often considered to be the main limitation in performing quantitative soot volume fraction measurements using LII $[1,12]$. In the present study, we 
aim at quantifying the effect of a varying laser sheet width on the obtained LII signal from 2D imaging of soot concentrations. We will show that the effect can be quite substantial as a result of two combined effects. First, the focusing creates a smaller probe volume at the focal plane in the centre of the imaged region. Second, while for a specific laser pulse energy, the whole interrogation region can give strong LII signal contribution, and at the focal plane, this may lead to strong sublimation, and thereby result in lower LII signal. Hence, the combined effect of a smaller probe volume and sublimation at the focal point in comparison with a spatial position outside the focal point can result in large variation in the LII signal and uncertainties in the quantification of soot volume fraction in a two - dimensional image.

The first reported study of the focusing effect investigated in this paper was by Shaddix and Smyth [13], who experimentally quantified the effect and used the experimentally observed LII signal variation across the flame to normalize LII signals from unsteady flame conditions. Although the effect was observed already in [13], no fundamental study including numerical modelling has been performed until recently. However, in this year, a study on the same topic was made by Shaddix and Williams [14], where they studied the spatial LII signal response to focusing using a single lens and single detection gate in a sooting diffusion flame, and supported the experimental work by numerical modelling work. Although our present study has a different approach, the main conclusions are similar.

In the present work, we used a one - dimensional flat flame in which a homogeneous soot field was created. Using different focal length lenses, we have demonstrated the effect of a varying sheet width in the imaged region. The experimental results were simulated using a heat-and-mass-transfer-based LII model [15] and good agreement was found. Using this model, uncertainties for various combinations of focal lengths and image lengths on the 2D LII signal were estimated. The results show that this effect can be substantial in 2D imaging experiments and this bias may, if not corrected for, compete with the uncertainty in the soot absorption efficiency on being the biggest uncertainty in quantification of LII signals in terms of soot volume fractions.

\section{Experimental setup}

Premixed ethylene-air flames $(\varphi=2.1)$ were set up on a McKenna burner as shown in Fig. 1a. A co-flow of air was introduced through the outer shroud of the burner and a metal plate was positioned on top at $21 \mathrm{~mm}$ height above burner (HAB) to stabilize the flame. A low-sooting flame $\left(\varphi=2.1\right.$, maximum $\left.f_{v}<60 \mathrm{ppb}\right)$ created a roughly onedimensional soot distribution, i.e. a radially homogeneous soot concentration at a certain HAB. LII measurements in

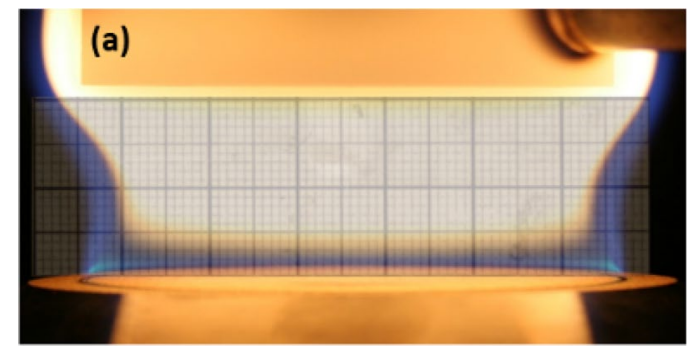

(b)

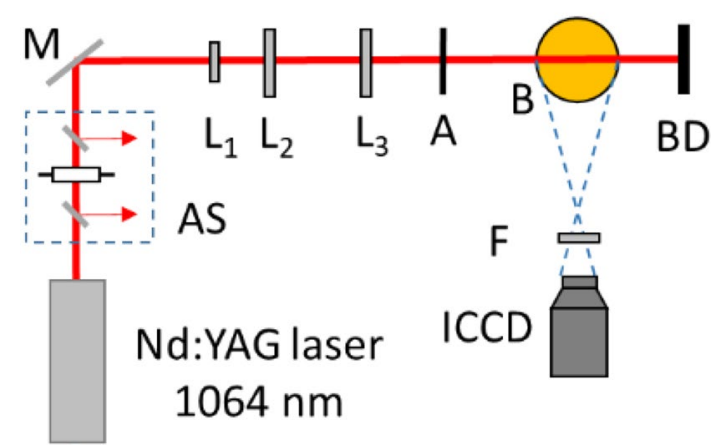

Fig. 1 a Premixed ethylene-air flame $(\varphi=2.1)$ stabilized on a McKenna burner. b Schematic representation of the optical setup. Acronyms: $A$ Aperture. $A S$ Attenuation System. $B$ Burner, $B D$ Beam Dump, $F$ Filter. L1, L2 and L3 - cylindrical lenses with focal lengths $-19 \mathrm{~mm},+200 \mathrm{~mm}$ and $+500 \mathrm{~mm}$

this flame gave strong LII signals and sufficiently low influence of laser energy attenuation along the beam path and signal trapping due to absorption by soot. At a height of $14 \mathrm{~mm}$ above burner, from which we show our main results, the extinction of the laser beam across the flame was $1.8 \%$, which is sufficiently low to be neglected in our further discussions. Additionally, the properties of the flame and the soot in this flame are well known from previous studies [16-18].

A schematic representation of the optical setup is shown in Fig. 1b. An Nd:YAG laser (Quantel Brilliant b) operated at $1064 \mathrm{~nm}(\mathrm{FWHM}=4 \mathrm{~ns})$ was used for the laser-induced incandescence (LII) measurements. The laser beam propagated through an attenuation system comprising of a halfwave plate placed in between two linear polarizers, which are aligned to transmit vertically polarized light and reflect horizontally polarized light. The half-wave plate in the middle can be used to rotate the polarization, thereby changing the vertical component of polarization and effectively the energy transmitted through the second linear polarizer. Using this system, the laser pulse energy can be varied in a wide range without changing the spatial energy profile of the laser. The laser beam with a diameter of around $5 \mathrm{~mm}$ was expanded vertically using a pair of cylindrical lenses ( $\mathrm{f}=-19 \mathrm{~mm}$ and $\mathrm{f}=+200 \mathrm{~mm}$ ). The vertical height was clipped to $16 \mathrm{~mm}$ using an aperture while maintaining the 
Gaussian spatial energy profile along the thickness direction. A cylindrical lens of focal length $+500 \mathrm{~mm}$ (denoted as f500 lens from now on) was used to focus the vertically extended laser beam into a sheet with the smallest beam waist at the centre of the burner. A beam profiling camera was used to estimate the thickness of the laser sheet at various locations along the direction of propagation, as discussed in Sect. 3.1. Two-dimensional LII signal intensities were acquired using a PIMAX 4 ICCD camera which was synchronized with the laser using a 'BNC Model 575' pulse generator (average jitter $=50 \mathrm{ps}$ ). The LII signal was collected at a wavelength of $445 \mathrm{~nm}$ employing a band-pass filter (central wavelength $445 \mathrm{~nm}$, FWHM $20 \mathrm{~nm}$ ) using four different gate widths, viz. $10 \mathrm{~ns}, 30 \mathrm{~ns}, 50 \mathrm{~ns}$ and $100 \mathrm{~ns}$. The start of the gate was set to a position $2 \mathrm{~ns}$ before the start of the LII signal. Background flame luminosities were also acquired semisimultaneously for all the gate widths and subtracted from the corresponding averaged LII images. At least 300 singleshot LII images were used for averaging.

\section{Methodology}

\subsection{Characterization of the propagating laser sheet}

Focusing of the laser beam using a cylindrical lens creates a variation in the sheet width near the focal plane. Laser sheet widths at various locations along the laser propagation direction were evaluated from the averaged 2D spatial distribution profiles of the laser sheet acquired using a beam profiling camera. After the sheet width measurements, the burner was mounted at the position where the minimum beam waist location coincides with the centre of the burner $(z=0)$. Laser sheet profiles in the width direction at the location of minimum sheet width $(z=0)$ and a location $20 \mathrm{~mm}$ away from this point along the laser beam direction $(z=20 \mathrm{~mm})$ are shown in Fig. 2a. The variation of the intensity along the height direction of the laser sheet was minimal and the profiles in Fig. 2a have been obtained by accumulating the signal intensity over a vertical height of $4 \mathrm{~mm}$. One can also observe the corresponding Gaussian fits in the same plots. The laser sheet width was evaluated as the width at $1 / \mathrm{e}^{2}(13.5 \%)$ of the maximum intensity. Using the evaluated sheet widths and pulse energy of the laser, the laser sheet profiles were converted to laser fluence profiles, as shown in Fig. 2a for a specific pulse energy of the laser. The variation of laser sheet thickness along the axial direction, $z$, is shown in Fig. 2b. Over a distance of $20 \mathrm{~mm}$ from $z=20$ to $z=0 \mathrm{~mm}$, the laser sheet thickness decreases from $299 \mu \mathrm{m}$ to $114 \mu \mathrm{m}$. Such a large variation of sheet thickness leads to a large variation of laser fluence as well along $z$, which is apparent by comparing the peak fluences at $z=0$ and $20 \mathrm{~mm}$ (Fig. 2a).
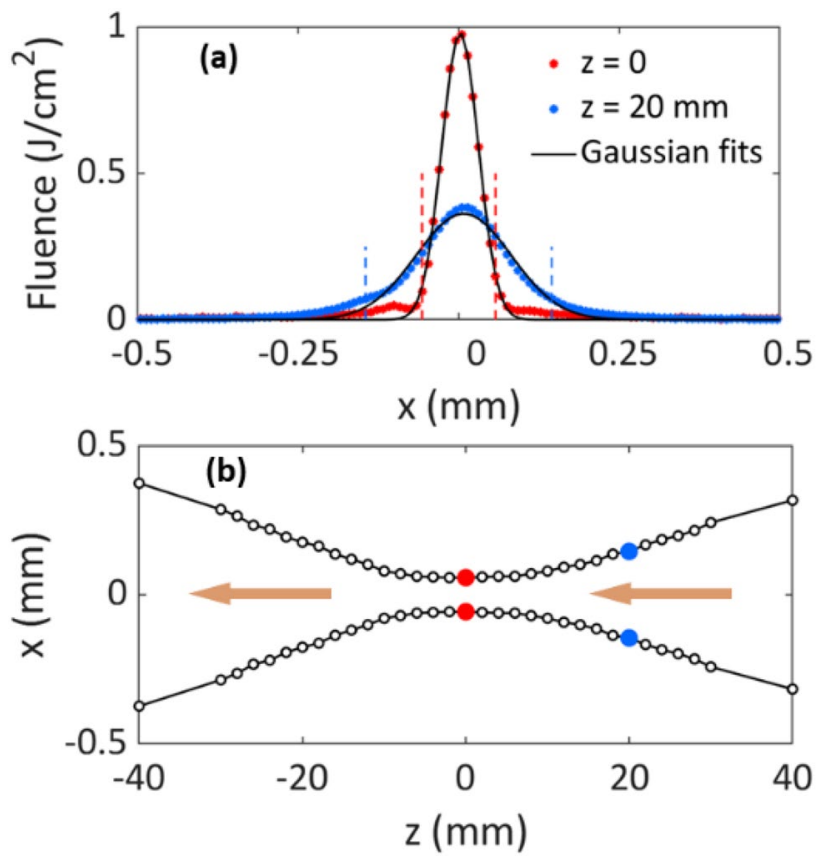

Fig. 2 a Laser fluence profile (case F5, Table 1) at $z=0$ and $z=20 \mathrm{~mm}$ where $\mathrm{z}$ is the direction of propagation of the laser. ' $x$ ' represents the dimension orthogonal to the laser sheet. Gaussian fits along with the evaluated sheet widths have also been marked. $\mathbf{b}$ Laser sheet profile along $z$. The locations of $z=0$ and $z=20 \mathrm{~mm}$ have been marked along with the direction of propagation of the laser

Table 1 Total pulse energies and the corresponding mean laser fluences at $z=0$ used for LII measurements

\begin{tabular}{lll}
\hline Symbol & Total pulse energy $(\mathrm{mJ})$ & $\begin{array}{l}\text { Mean fluence } \\
\text { at } z=0\left(\mathrm{~J} / \mathrm{cm}^{2}\right)\end{array}$ \\
\hline F1 & 2.24 & 0.12 \\
F2 & 4.48 & 0.24 \\
F3 & 6.72 & 0.37 \\
F4 & 8.96 & 0.49 \\
F5 & 11.2 & 0.61 \\
F6 & 13.4 & 0.73 \\
F7 & 15.7 & 0.86 \\
F8 & 17.9 & 0.98 \\
F9 & 20.2 & 1.10 \\
F10 & 22.4 & 1.22 \\
\hline
\end{tabular}

Hence, during the focusing of a pulse (with constant pulse energy), a broader sheet with lower peak fluence at $z=20 \mathrm{~mm}$ transfers to a thinner sheet with higher peak fluence at $z=0 \mathrm{~mm}$. LII measurements were performed for a wide range of laser pulse energies, as shown in Table 1, along with the corresponding laser fluences at $z=0$ for all the measurement cases. 


\subsection{LII model}

A heat-and-mass-transfer-based LII model [15] was employed to model the experimental LII. For a specific fluence case in Table 1 (a specific total laser pulse energy), Gaussian distributions of the laser energy (or fluence) were generated using the sheet width at each point along the laser propagation direction, $z$ (Fig. $2 b$ ). The spatial region containing the laser is divided into equidistant bins (bin width $\sim 2.3 \mu \mathrm{m}$ ) at each $z$ location. This is done to take the probe volume into account for the LII model evaluation where the number of bins is proportional to the sheet width. LII signals were calculated for each of these bins (with corresponding fluences) and added up to get the total LII signal at each specific $z$-position. At least 50 bins were used for each $z$ location, and a uniform spatial distribution of soot was assumed in the probed region. The relevant parameters used for the LII model evaluation are given in Table 2. Previous studies of the same flame provided values for various parameters used as input data in the model; flame temperature [16], primary particle diameter [17] and the absorption function, $E(m)$ [18].

Figure 3 shows time-resolved LII signals simulated by the LII model for the location of minimum laser sheet thickness $(z=0)$ and $z=20 \mathrm{~mm}$ for the fluence case F5. It can be seen that the peak signal at $z=0$ is lower compared to that at $z=20 \mathrm{~mm}$. There are two main factors which come into play here. First, the mean fluence is $0.61 \mathrm{~J} / \mathrm{cm}^{2}$ at $z=0$ which is much higher compared to $0.23 \mathrm{~J} / \mathrm{cm}^{2}$, the value at $z=20 \mathrm{~mm}$ due to the larger sheet thickness at $z=20 \mathrm{~mm}$. Such a high value of fluence at $z=0$ causes a much higher sublimation, which leads to lower LII signal and earlier LII peak position. The second factor is the larger probe volume at $z=20 \mathrm{~mm}$ (wider sheet) compared to that at $z=0$, resulting in lot more soot particles being illuminated by the laser. Although the fluence at $z=20$ is lower than that at $z=0$, it is high enough to produce incandescence. Hence, a combination of these two factors produces a larger total peak signal intensity at $z=20 \mathrm{~mm}$ as seen in Fig. 3. If one analyses the decay rates of the signals, the differences due to soot sublimation can also be seen as the decay is much faster at $z=0$ with stronger

Table 2 LII model parameters

\begin{tabular}{lll}
\hline No & Parameter & Value/model used \\
\hline 1 & Flame temperature & $1595 \mathrm{~K}[16]$ \\
2 & Primary particle diameter & $12.5 \mathrm{~nm}[17]$ \\
3 & Number of particles per aggregate & 1 \\
4 & Soot absorption function, $E(m)$ & $0.35[18]$ \\
5 & Thermal accommodation coefficient, $\alpha_{T}$ & $0.37[3]$ \\
6 & Mass accommodation coefficient, $\beta$ & $0.8[19]$ \\
7 & Detection wavelength & $445 \mathrm{~nm}$ \\
\hline
\end{tabular}

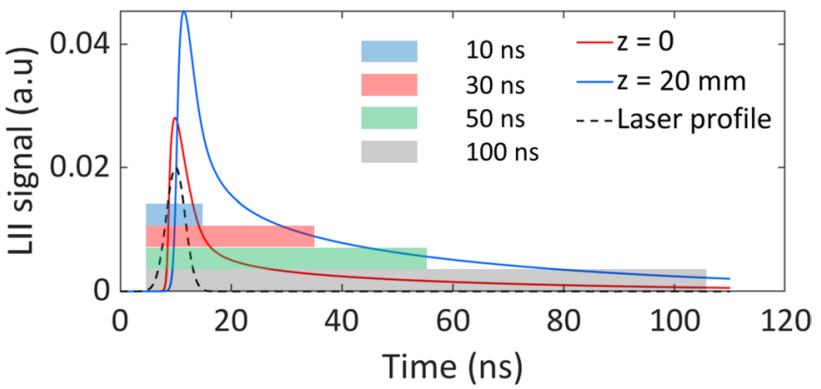

Fig. 3 Time-resolved LII signals calculated using the LII model at the minimum beam waist location $(z=0)$ and at $z=20 \mathrm{~mm}$ for the case with the fluence F5. The various gate widths used for analysis is marked along with the laser temporal profile

particle sublimation. The gate widths and gate locations used in the present study are also shown in Fig. 3.

Figure 4 shows the variation of the gated experimental and modelled LII signals (gate width $=30 \mathrm{~ns}$ ) at $14 \mathrm{~mm} \mathrm{HAB}$ with laser pulse energy at the locations $z=0,10$ and $20 \mathrm{~mm}$. For the measurement cases (Fig. 4a), the start of the gate was set to a position $2 \mathrm{~ns}$ before the start of the LII signal. Meanwhile, in the model (Fig. 4b), the gate starts $5 \mathrm{~ns}$ before the peak of the laser pulse. In Fig. 4, it can be seen that at lower pulse energies, for example, $4.5 \mathrm{~mJ}$ (case F2), the LII signal is stronger at $z=0$ compared to $z=20 \mathrm{~mm}$. Here, the larger sheet width at $z=20 \mathrm{~mm}$ leads to a much lower laser fluence, thereby producing weaker incandescence signals.
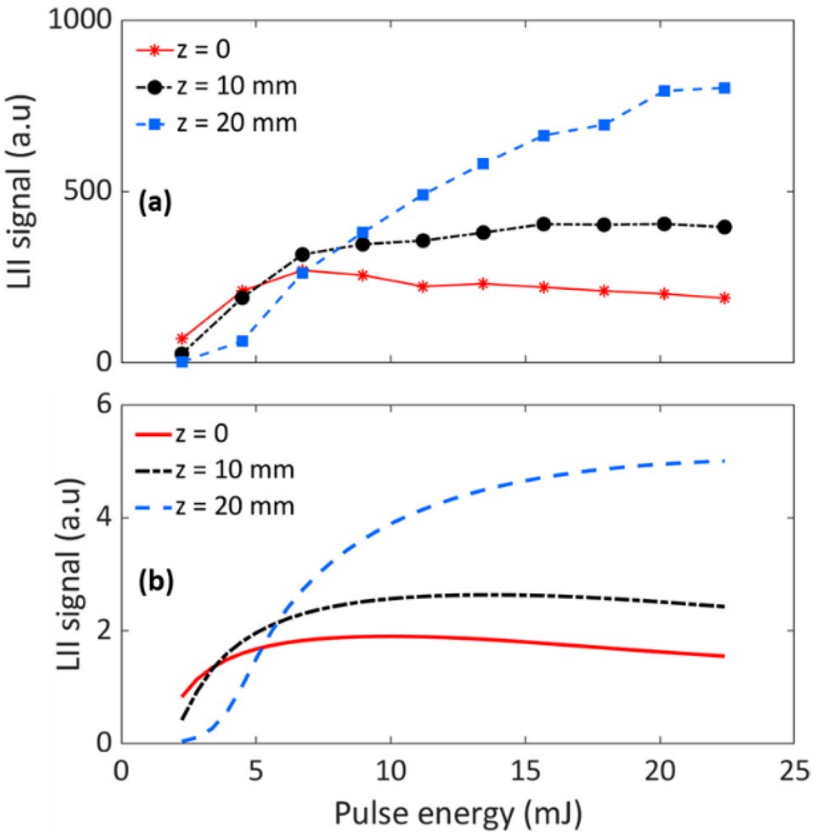

Fig. 4 LII signal variation with laser pulse energy at $z=0,10$ and $20 \mathrm{~mm}$ using a $f=500 \mathrm{~mm}$ lens at $14 \mathrm{~mm}$ HAB obtained from both (a) LII measurements and (b) LII model 
As the pulse energy increases, soot at $z=0$ starts to sublime before that at $z=20 \mathrm{~mm}$. The added effect of larger probe volume along with the lower sublimation levels results in stronger LII signals at $z=20 \mathrm{~mm}$ compared to $z=0$. It can also be seen that from a pulse energy of $11.2 \mathrm{~mJ}$ (case F5) and above using an $f=500 \mathrm{~mm}$ lens, there is an increase in the LII signal intensity by a factor of at least two over a distance of $20 \mathrm{~mm}(0-20 \mathrm{~mm})$, thus creating a drastic uncertainty in the estimated soot volume fraction from a 2D imaging experiment. It can also be seen that the experimental and modelled signals match very well.

\section{Results}

To demonstrate the influence of the focal length of the focusing lens, Fig. 5a, b show the 2D LII signal intensity distribution for two different cases in the investigated flame. The two cases are an f2000 lens (Fig. 5a) and an f500 lens (Fig. 5b), and the measurements were performed using a fluence of $0.49 \mathrm{~J} / \mathrm{cm}^{2}$ at $z=0$ (F4 in Table 1) and camera gate width $50 \mathrm{~ns}$. LII signal profiles at $14 \mathrm{~mm}$ HAB for the cases using the corresponding lenses are also plotted in Fig. 5c, $d$ for various gate widths of the camera. It can be seen from Fig. $5 \mathrm{c}$ that for the f2000 lens, the LII signal between $z=-20 \mathrm{~mm}$ and $z=20 \mathrm{~mm}$ have fairly constant signal intensities. However, the signal profiles for the case using the f500 lens shown in Fig. 5d show a large variation over the same distance. To investigate this behaviour in detail, a region between $z=-6 \mathrm{~mm}$ and $z=20 \mathrm{~mm}$ was selected for further analysis.

Gaussian distributions of laser fluence were generated at locations between $z=-6 \mathrm{~mm}$ and $z=20 \mathrm{~mm}$ using the corresponding sheet widths from Fig. $2 \mathrm{~b}$ as described in Sect. 3.2. LII model evaluations were performed for these radial locations for all the fluence cases ranging from $\mathrm{F} 1$ to

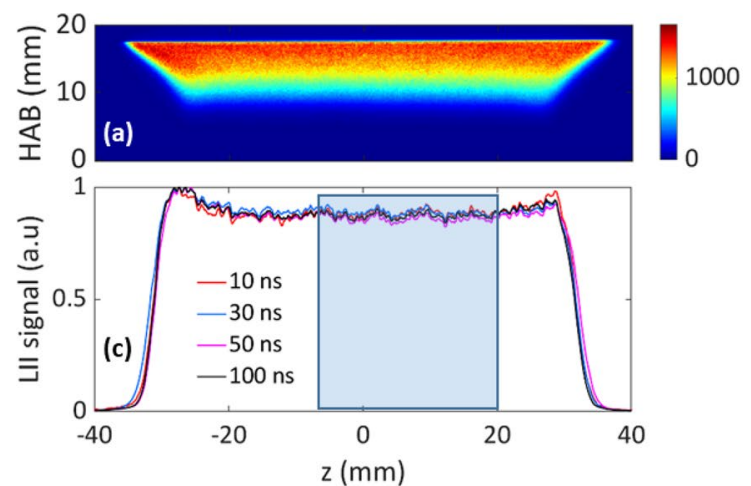

Fig. 5 2D LII signal intensity distributions using lenses with focal lengths (a) $2000 \mathrm{~mm}$ and (b) $500 \mathrm{~mm}$. Both the images are acquired for the case with fluence F4 and a camera gate width of $50 \mathrm{~ns}$. The colour bar represents LII signal intensity (a.u). Corresponding LII
F10 while using $10 \mathrm{~ns}, 30 \mathrm{~ns}, 50 \mathrm{~ns}$ and $100 \mathrm{~ns}$ as the gate widths of signal acquisition. Figure 6 shows both the measurement and modelled LII data for some selected fluences. A first observation in the figure is that there is a significant variation in the LII signal at all fluences in this spatial range. Ideally, the region between $z=-6 \mathrm{~mm}$ and $z=20 \mathrm{~mm}$ shown in the figure should produce fairly constant LII signal intensities as seen in Fig. 5c, for which an f2000 lens was used to focus the laser sheet. In the present flame, laser beam attenuation and signal trapping can be considered negligible as the mean soot volume fraction at $14 \mathrm{~mm} \mathrm{HAB}$ is $\sim 40 \mathrm{ppb}$ [4] resulting in a net extinction of $\sim 1.8 \%$. This implies that the difference in the LII signal between $z=-6 \mathrm{~mm}$ and $z=20 \mathrm{~mm}$ should be mainly due to the variation in the laser sheet width and consequently the variation in fluence. It can also be seen that the experimental and modelled curves in Fig. 6 match quite well at all fluences.

If one analyses the case F1 in Fig. 6, it can be seen that the normalised signal is highest at $z=0$, and at $z=20 \mathrm{~mm}$, the signal is less than $1 / 10$ th of the maximum. Here, as the mean fluence near $z=20 \mathrm{~mm}$ is too low to produce significant incandescence, only the central region of the burner gives strong LII signals. For the fluence case F3, soot at $z=20 \mathrm{~mm}$ also starts to produce strong incandescence signals. The added effects of a larger probe volume and lower sublimation compared to $z=0$ make the signal at $z=20 \mathrm{~mm}$ approximately 1.5 times stronger (LII model, F3, 30 ns case) than that at $z=0$. For the fluence cases F5 and higher, the effect of sublimation becomes even more strong at $z=0$ resulting in producing a signal at $z=20 \mathrm{~mm}$ which is $\sim 2.2$ times stronger (LII model, F5, $30 \mathrm{~ns}$ case) than that at $z=0$.

Another interesting observation in Fig. 6 is the behaviour of LII signals acquired using different gate widths. It can be seen that for fluences ranging from F3 to F10, the $10 \mathrm{~ns}$ case shows the lowest variation compared to the other gate widths. This is due to the effect of soot sublimation, which

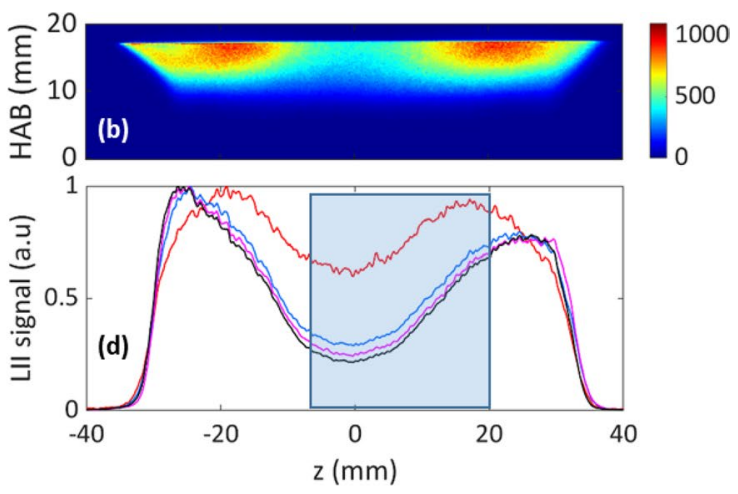

signal intensity profiles at $14 \mathrm{~mm} \mathrm{HAB}$ at various gate widths are shown in (c) and (d), respectively. The region used for further analysis ranging from $z=-6$ to $20 \mathrm{~mm}$ has also been marked 

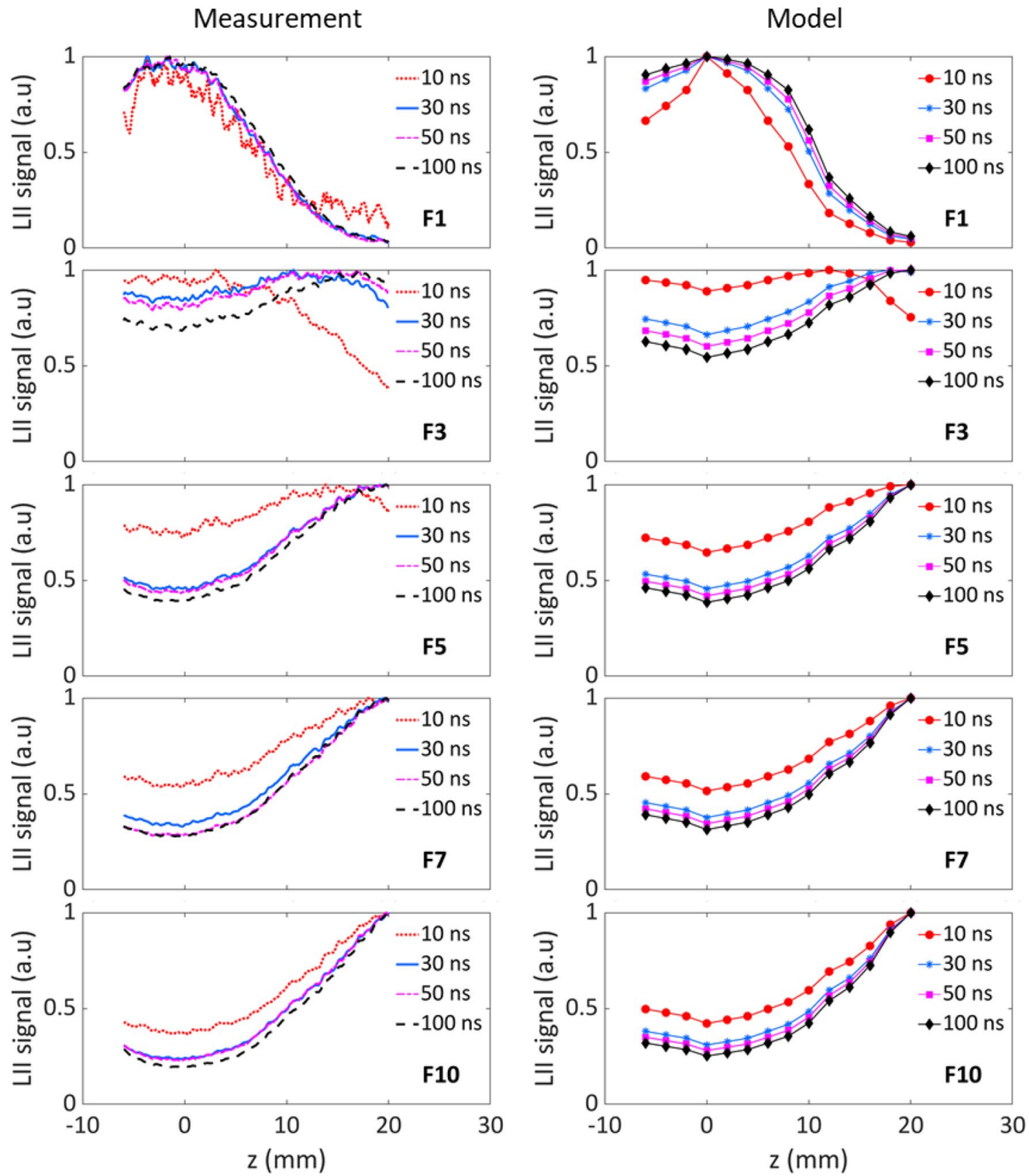

Fig. 6 LII signal intensities at 14 mm HAB generated using measurements (left) and the LII model (right) for the fluence cases F1, F3, F5, F7 and $\mathrm{F} 10$ at acquisition gate widths of $10 \mathrm{~ns}, 30 \mathrm{~ns}, 50 \mathrm{~ns}$ and $100 \mathrm{~ns}$. All signal curves are normalized to the highest intensity

reduces the peak LII signal and increases the LII signal decay rate. The effect of decay rate for a short gate width like $10 \mathrm{~ns}$ is not as significant as that for the longer gate widths and, hence, the spatial LII signal variation is larger for longer gate widths.

From both the experimental and modelled LII signal profiles at $14 \mathrm{~mm}$ HAB shown in Fig. 5, it can be seen that the f500 lens is not suitable for studying the present flame. To understand the behaviour of other lenses towards the same problem, the variations of laser sheet thickness along the direction of laser beam propagation have been modelled using a Gaussian beam propagation model [20] for the lenses with focal lengths of $500 \mathrm{~mm}, 1000 \mathrm{~mm}$ and $2000 \mathrm{~mm}$. The modelled sheet thickness variations along $z$ are shown in Fig. 7. This model required the laser wavelength and initial beam waist as the main input parameters. An initial laser beam waist thickness of $7.5 \mathrm{~mm}$ provided a fairly good match for the experimental and modelled beam waists for 


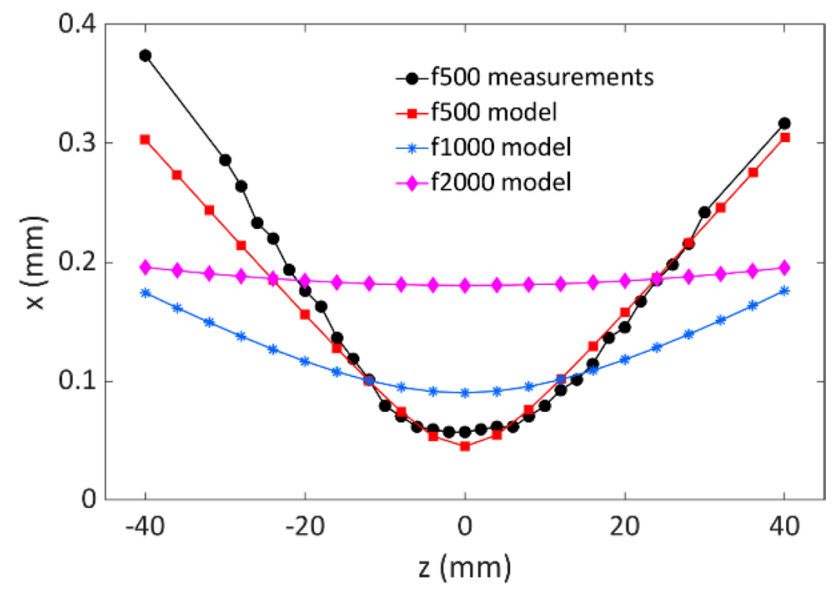

Fig. 7 Experimental and modelled laser sheet thickness profiles $(x)$ along the direction of propagation $(z)$ for the f500 lens. Modelled laser sheet thickness profiles for $\mathrm{f} 1000$ and $\mathrm{f} 2000$ have also been plotted

the case of the 5500 lens. The same value was used for modelling the laser sheet propagation for the $\mathrm{f} 1000$ and $\mathrm{f} 2000$ lenses. It can be seen from Fig. 7 that the 1000 lens has a much lower variation in the sheet thickness compared to the f500 lens. The sheet widths at $z=40$ for the 500 and the f1000 lenses are, respectively, 6.7 and 2.0 times larger than their corresponding values at $z=0$. Meanwhile, the f2000 lens showed negligible variation in the sheet width as this factor is only 1.1. Obviously, this shows that using lenses with longer focal lengths considerably improve the spatial distribution of the sheet width in the measurement region.

Based on the good agreement between experimental and modelled data shown in Fig. 6, the LII model can be used to estimate optimal experimental conditions to achieve the same relationship between LII signal and soot volume fraction over the whole 2D surface. To demonstrate this, LII model evaluations have been performed for the $\mathrm{f} 1000$ lens and the resulting signal profiles are shown in Fig. 8. Model evaluations were performed for various fluences for all the four gate widths used before. Also, note that Fig. 8 deals with double the range of $z(80 \mathrm{~mm})$ as compared to Fig. $6(40 \mathrm{~mm})$. A first interesting observation in Fig. 8 is that the variation in the LII signal across $\mathrm{z}$ for a specific range of $\mathrm{z}$ is much lower compared to the corresponding fluence cases using the f500 lens shown in Fig. 6. As an example, for the case F5 with gate width $30 \mathrm{~ns}$, the signal at $z=20 \mathrm{~mm}$ is $\sim 2.2$ times stronger than that at $z=0$ for the f500 lens (Fig. 6). For the f1000 lens, shown in Fig. 8, this factor is only $\sim 1.3$ showing that using lenses with longer focal lengths, the variation in the LII signal along $z$ can be reduced significantly. Meanwhile, for the same case using the $\mathrm{f} 1000$ lens, the signal further away at $z=40 \mathrm{~mm}$ is $\sim 1.9$ times stronger than that at $z=0$. This suggests that if one
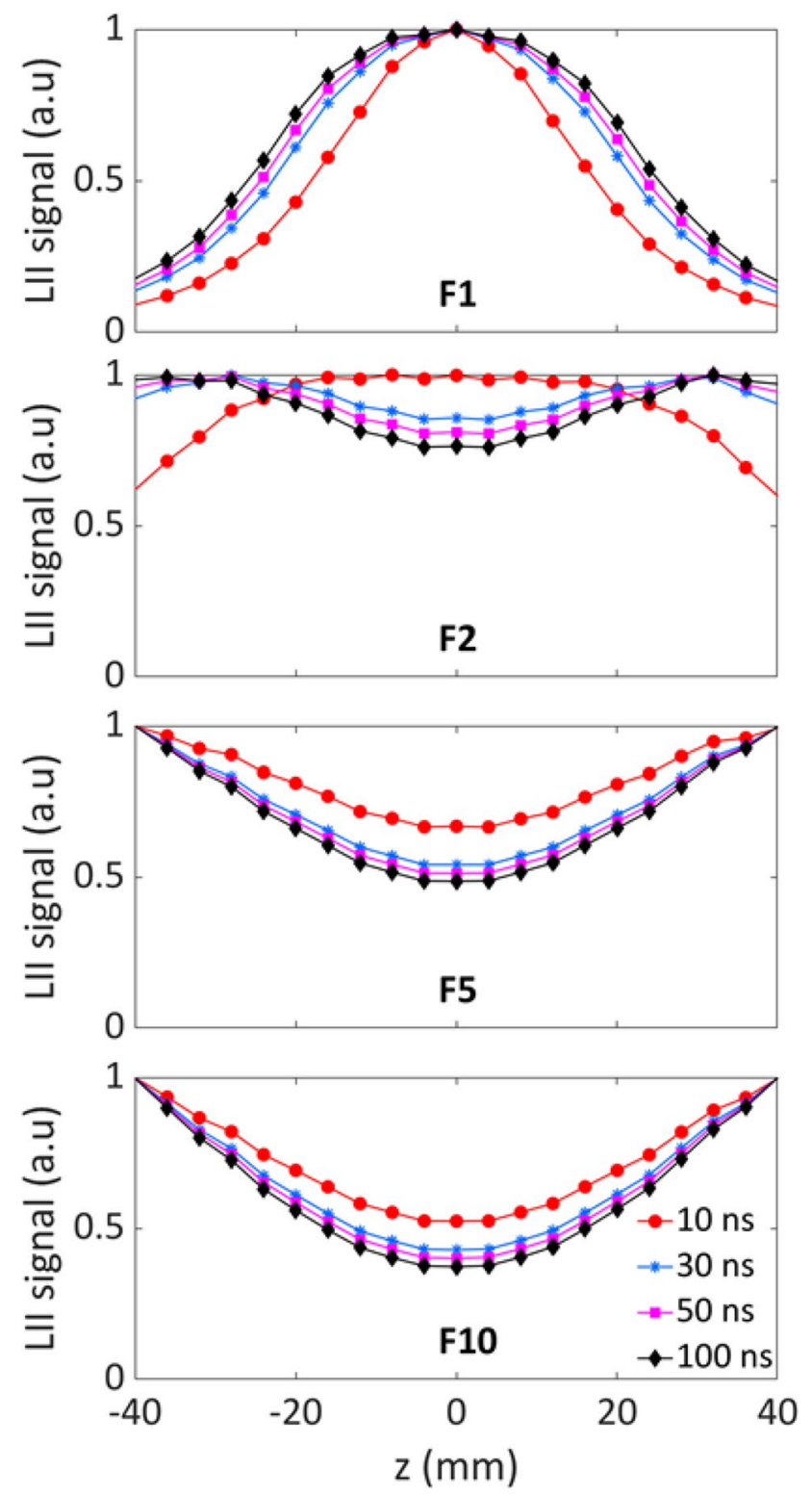

Fig. 8 The variation of LII signal profiles evaluated using the LII model for the f1000 lens along the laser propagation direction $(z)$. The laser sheet thicknesses along $z$ was obtained using a Gaussian beam propagation model. The plots correspond to various fluence cases F1, F2, F5 and F10

wishes to keep this factor below a certain value or in other words, keep the variation in the LII signal response to soot volume fraction below a certain factor in an image, the data analysis has to be restricted to a certain region around $z=0$ in the beam propagation direction. We denote this distance as image length (IL). Portraying this idea in another perspective, accurate LII measurements for a certain image length would require a focusing lens with focal lengths above a certain value. It is also noteworthy that the fluence cases in Fig. 8 resemble the trends observed in another study done 
on the effects of laser sheet focusing while using an f1000 lens [14].

The LII model can also be effectively used for deciding the focal length of the lens required to perform accurate LII measurements (i.e., with low uncertainty in LII signal response to soot volume fraction in a 2D image) for a certain image length. Based on the image length, Fig. 9 suggests a way to select primarily the focal length of the focusing lens, among other measurement parameters, such as the laser fluence and detection gate width. The $\mathrm{x}$-axis in the plots represent the ratio of focal length (FL) of the focusing lens and the image length (FL/IL ratio). The y-axis represents the ratio between the LII signals at $z=z^{\prime}$ and $z=0$ (referred to as 'signal ratio') where, $2 z^{\prime}$ represents the image length. The closer the signal ratio is to 1 , the lower is the uncertainty in the corresponding LII measurement due to variation in the sheet width.
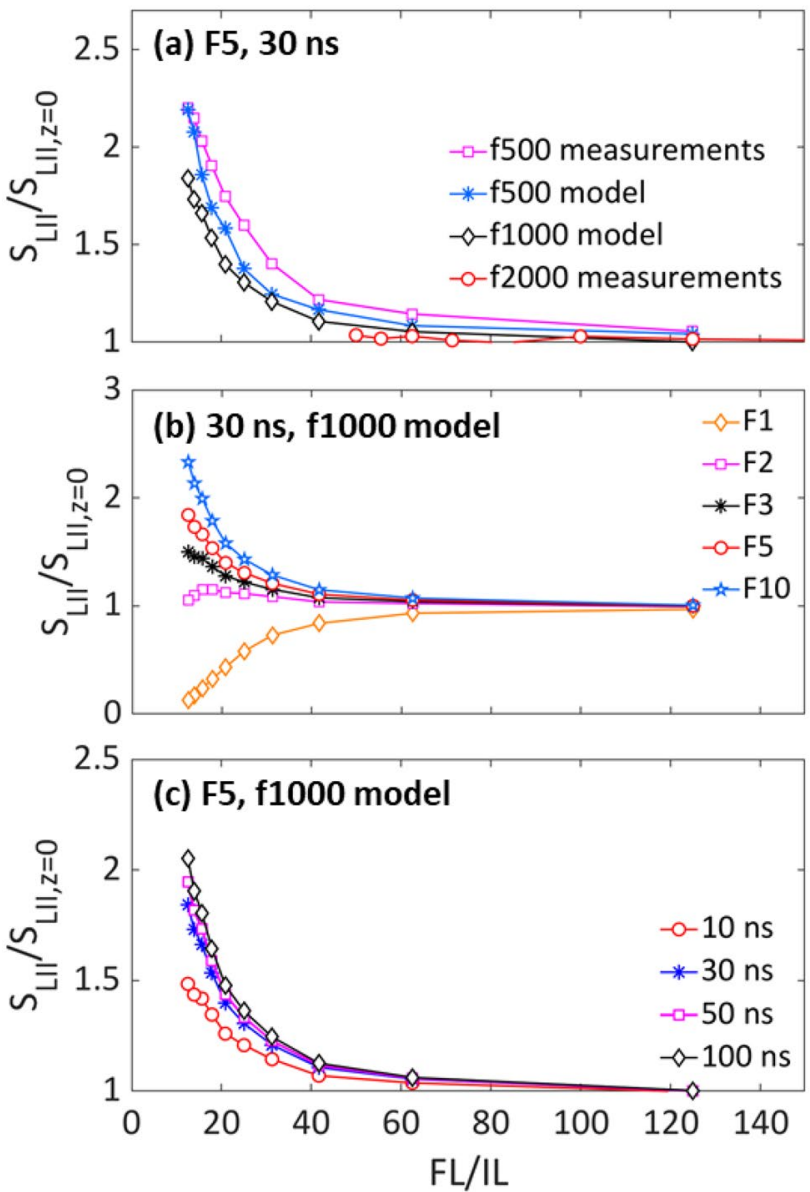

Fig. 9 Variation of signal ratio (ratio between the LII signals at $z=z$ ' and $z=0$ ) with the ratio between the focal length (FL) of the focusing lens and the image length (IL)for: (a) various lenses for the fluence case F5 and detection gate of $30 \mathrm{~ns}$. b various fluences for the case of modelled f1000 lens with detection gate of $30 \mathrm{~ns}$. c various detection gate widths for the case of modelled f1000 lens for the fluence case F5
Figure 9a shows the variation of the signal ratio with the FL/IL ratio for both the measurement and model cases using various focusing lenses (case F5, 30 ns gate width). In this plot, $z^{\prime}$ ranges from 2 to $20 \mathrm{~mm}$ resulting in the variation of image lengths from 2 to $40 \mathrm{~mm}$ for all the cases other than the $\mathrm{f} 1000$ model where the largest image length is $80 \mathrm{~mm}$. A first observation in Fig. 9a is that the signal ratio starts to increase rapidly when the FL/IL ratio is lowered below a certain value for all the cases. One can also compare the signal ratios for a specific image length for different lenses. For example, for an image length of $24 \mathrm{~mm}$, the signal ratios while using an f500 lens (FL/IL $=21)$ and f1000 lens (FL/ $\mathrm{IL}=42$ ) are 1.6 and 1.1, respectively. (Here, we should remind that a factor of 1.6 means that there is a $60 \%$ difference in LII signal response for a constant soot volume fraction across the image). The measured and model curves for f500 are in fair agreement, and it is not surprising that the measured values for the signal ratio are slightly higher due to experimental uncertainties, such as flame homogeneity and signal noise. We can also observe that the modelled signal ratios for longer focal length, f1000 in comparison with f500, are slightly lower. The model we have created can now be used to estimate any combination of focal length and image length. As an example, if a bias in LII signal of, let us say, $10 \%$ is accepted over a chosen image length in a measurement situation, the presented curves can be used to calculate the minimum focal length that is needed to fulfil this requirement. Additionally, the experimental uncertainty has to be added. Once a lens with an appropriately long focal length has been selected, the measurement uncertainties can be further reduced by careful selection of laser fluence and detection gate as shown in Fig. 9b, c.

Figure $9 \mathrm{~b}$ shows the variation of the signal ratio with the FL/IL ratio for different fluence cases while using a f1000 lens and a detection gate width of 30 ns. Fluence case F1 shows large uncertainties for large image lengths (short FL/ IL ratios). However, the low fluence case F2 shows signal ratios close to 1 for much larger image lengths compared to the cases F3 and F5 with higher fluence. Low fluence cases might be risky for LII measurements in systems with large image lengths due to laser beam attenuation by soot absorption. A drop in laser pulse energy combined with the effects of variation of sheet width along $z$ might lead the fluence cases F2 to show trends similar to F1. Meanwhile, cases with too high fluence, such as F10, also cause large bias in LII signal intensities for larger image lengths. Consequently, an optimal fluence has to be selected based on the actual measurement situation to minimize the LII signal bias across the 2D image.

In Fig. 9c, the effect of detection gate widths on the signal ratio is analysed. It can be seen that smaller gate widths showed lower bias in LII signal intensity for large image lengths. Hence, for a chosen focal length of the lens, the 
LII signal variation along the image length can be minimized using a short detection gate width and an optimal laser fluence.

An analysis of the impact of uncertainties involved with the flame and soot properties in the resulting signal ratios was also performed using the LII model. The fluence case F5 with 30 ns gate width was used for this analysis. It was found that individual variations as high as $20 \%$ in flame temperature, primary particle size and soot absorption function resulted in variations in the signal ratio which are less than $3 \%, 0.5 \%$ and $8 \%$, respectively. Hence, in comparison with these factors, the choice of FL/IL ratio in an experiment will generally have a larger impact on the uncertainty in soot concentration measurements if not corrected for.

It seems that two-dimensional LII-based studies performed in large systems often have underestimated the uncertainties in the LII signal caused due to variation in laser sheet width along the direction of propagation, and this issue is rarely discussed as a main uncertainty in quantification of 2D LII signals in terms of soot volume fractions. There are several studies which were performed with combinations of focal lengths of the focusing lens and image lengths resulting in low FL/IL ratios [8, 21-23]. For example, one of the investigations used an $\mathrm{f} 600$ lens for an image length of $40 \mathrm{~mm}$ which results in an FL/IL ratio of 15 [22]. Another study, used a combination of f500 lens and an image length of $30 \mathrm{~mm}$ resulting in an FL/IL ratio of $\sim 17$ [21]. Although the 2D LII images were not used for any quantitative analysis, a study performed in an oxy-fuel reactor involved an f2000 lens and an image length of $150 \mathrm{~mm}$ used an FL/IL ratio of $\sim 13$ [8]. Although these studies do not have exactly the same conditions as the present investigation, it can be seen from Fig. 9 that such low FL/IL ratios could result in significant uncertainties in the 2D LII signal distribution. These uncertainties get carried forward to the calibrated soot volume fraction distributions, usually obtained using extinction measurements as the calibration technique, which is unaffected by the laser beam width variation. This study, thus, spreads light into the significance of this uncertainty due to varying laser sheet width in 2D LII-based investigations of soot and recommends ways to mitigate these effects.

\section{Conclusions}

In 2D LII imaging measurements where the laser sheet is focused at the measurement region using a cylindrical lens, the variation of sheet width along the measurement region creates uncertainties in the resulting 2D LII signal image. The effects of the variation of sheet width and the resulting uncertainties have been studied using LII measurements and a heat-and-mass-transfer-based LII model. LII measurements were performed on premixed ethylene-air flames ( $\varphi=2.1)$ stabilized on a McKenna burner, where other uncertainties, such as laser beam attenuation and signal trapping, are negligible. The following conclusions have been formulated from this study:

1. The bias in the 2D LII signal resulting from the variation of laser sheet width in the measurement region was found to be significant. This bias can easily reach several tens of percent, thereby competing with uncertainties in soot absorption properties in being the dominating source of uncertainty when quantification of LII signals is done in terms of soot volume fraction.

2. Numerical calculations using a heat-and-mass-transferbased LII model showed good agreement with experiments, and it was used to estimate LII signals across 2D LII images for various combinations of focal lengths, image lengths, gate widths and laser fluences.

3. The ratio between the focal length (FL) of the focusing lens and the image length (IL), i.e., the FL/IL ratio, was introduced as a valuable parameter to estimate the LII signal response to soot volume fraction over the image length. It was demonstrated that high FL/IL ratios minimize this bias, which practically means to strive to use a very long focal length of the focusing lens.

4. Additionally, for a chosen focal length of the focusing lens, the uncertainties due to variation of sheet width can be minimized using a short detection gate width and an optimal choice of laser fluence for each specific measurement situation.

Acknowledgements This project was financially supported by the Swedish Energy Agency through the Swedish Gasification Centre (SFC) (34721-3), as well as from the European Union's Horizon 2020 research and innovation programme under the Marie Sklodowska-Curie grant agreement No 675528 .

Funding Open access funding provided by Lund University.

Open Access This article is licensed under a Creative Commons Attribution 4.0 International License, which permits use, sharing, adaptation, distribution and reproduction in any medium or format, as long as you give appropriate credit to the original author(s) and the source, provide a link to the Creative Commons licence, and indicate if changes were made. The images or other third party material in this article are included in the article's Creative Commons licence, unless indicated otherwise in a credit line to the material. If material is not included in the article's Creative Commons licence and your intended use is not permitted by statutory regulation or exceeds the permitted use, you will need to obtain permission directly from the copyright holder. To view a copy of this licence, visit http://creativecommons.org/licenses/by/4.0/. 


\section{References}

1. H. Michelsen, C. Schulz, G. Smallwood, S. Will, Laser-induced incandescence: particulate diagnostics for combustion, atmospheric, and industrial applications. Prog Energy Combust Sci 51, 2-48 (2015)

2. C. Schulz, B.F. Kock, M. Hofmann, H. Michelsen, S. Will, B. Bougie, R. Suntz, G. Smallwood, Laser-induced incandescence: recent trends and current questions. Appl Phys B 83(3), 333 (2006)

3. H. Bladh, J. Johnsson, P.-E. Bengtsson, On the dependence of the laser-induced incandescence (LII) signal on soot volume fraction for variations in particle size. Appl. Phys. B 90(1), 109-125 (2008)

4. J. Simonsson, N.-E. Olofsson, S. Török, P.-E. Bengtsson, H. Bladh, Wavelength dependence of extinction in sooting flat premixed flames in the visible and near-infrared regimes. Appl Phys B 119(4), 657-667 (2015)

5. Z. Sun, Z. Alwahabi, D. Gu, S. Mahmoud, G. Nathan, B. Dally, Planar laser-induced incandescence of turbulent sooting flames: the influence of beam steering and signal trapping. Appl Phys B 119(4), 731-743 (2015)

6. J. Zerbs, K. Geigle, O. Lammel, J. Hader, R. Stirn, R. Hadef, W. Meier, The influence of wavelength in extinction measurements and beam steering in laser-induced incandescence measurements in sooting flames. Appl Phys B 96(4), 683-694 (2009)

7. E. Huestis, P.A. Erickson, M.P. Musculus, In-cylinder and exhaust soot in low-temperature combustion using a wide-range of EGR in a heavy-duty diesel engine. SAE Trans 1, 860-870 (2007)

8. J. Simonsson, A. Gunnarsson, M.N. Mannazhi, D. Bäckström, K. Andersson, P.-E. Bengtsson, In-situ soot characterization of propane flames and influence of additives in a $100 \mathrm{~kW}$ oxy-fuel furnace using two-dimensional laser-induced incandescence. Proc Combust Inst 37(1), 833-840 (2019)

9. K. Frederickson, S.P. Kearney, T.W. Grasser, Laser-induced incandescence measurements of soot in turbulent pool fires. Appl Opt 50(4), A49-A59 (2011)

10. J. Reimann, S.-A. Kuhlmann, S. Will, 2D aggregate sizing by combining laser-induced incandescence (LII) and elastic light scattering (ELS). Appl Phys B 96(4), 583-592 (2009)

11. N. Hashimoto, J. Hayashi, N. Nakatsuka, K. Tainaka, S. Umemoto, H. Tsuji, F. Akamatsu, H. Watanabe, H. Makino, Primary soot particle distributions in a combustion field of $4 \mathrm{~kW}$ pulverized coal jet burner measured by time resolved laser induced incandescence (TiRe-LII). J Therm Sci Technol 11(3), 49 (2016)

12. F. Liu, J. Yon, A. Fuentes, P. Lobo, G.J. Smallwood, J.C. Corbin, Review of recent literature on the light absorption properties of black carbon: Refractive index, mass absorption cross section, and absorption function. Aerosol Sci Technol 54(1), 33-51 (2020)

13. C.R. Shaddix, K.C. Smyth, Laser-induced incandescence measurements of soot production in steady and flickering methane, propane, and ethylene diffusion flames. Combust Flame 107(4), 418-452 (1996)

14. C.R. Shaddix, T.C. Williams, Analysis of laser focusing effect on quantification of LII images. Proc Combust Inst 7, 8 (2020)

15. H. Bladh, P.-E. Bengtsson, Characteristics of laser-induced incandescence from soot in studies of a time-dependent heat-and masstransfer model. Appl. Phys. B 78(2), 241-248 (2004)

16. N.-E. Olofsson, J. Johnsson, H. Bladh, P.-E. Bengtsson, Soot sublimation studies in a premixed flat flame using laser-induced incandescence (LII) and elastic light scattering (ELS). Appl Phys B 112(3), 333-342 (2013)

17. H. Bladh, J. Johnsson, N.-E. Olofsson, A. Bohlin, P.-E. Bengtsson, Optical soot characterization using two-color laser-induced incandescence (2C-LII) in the soot growth region of a premixed flat flame. Proc Combust Inst 33(1), 641-648 (2011)

18. N.-E. Olofsson, J. Simonsson, S. Török, H. Bladh, P.-E. Bengtsson, Evolution of properties for aging soot in premixed flat flames studied by laser-induced incandescence and elastic light scattering. Appl Phys B 119(4), 669-683 (2015)

19. DR Snelling, F Liu, GJ Smallwood, ÖL Gülder (2000) Evaluation of the nanoscale heat and mass transfer model of LII: prediction of the excitation intensity. Proceedings of the 34th National Heat Transfer Conference, Pittsburg, PA.

20. S.A. Self, Focusing of spherical Gaussian beams. Appl Opt 22(5), 658-661 (1983)

21. S. Balusamy, M.M. Kamal, S.M. Lowe, B. Tian, Y. Gao, S. Hochgreb, Laser diagnostics of pulverized coal combustion in $\mathrm{O} 2 / \mathrm{N} 2$ and $\mathrm{O} 2 / \mathrm{CO} 2$ conditions: velocity and scalar field measurements. Exp Fluids 56(5), 108 (2015)

22. M. Köhler, K. Geigle, W. Meier, B. Crosland, K. Thomson, G. Smallwood, Sooting turbulent jet flame: characterization and quantitative soot measurements. Appl Phys B 104(2), 409-425 (2011)

23. C.R. Shaddix, T.C. Williams, The effect of oxygen enrichment on soot formation and thermal radiation in turbulent, non-premixed methane flames. Proc Combust Inst 36(3), 4051-4059 (2017)

Publisher's Note Springer Nature remains neutral with regard to jurisdictional claims in published maps and institutional affiliations. 